\title{
LE LAIt D'HIER ET LE LAIT DE DEMAIN (1)
}

\author{
par \\ G. THIEULIN et A. HOUDINIÈRE
}

Les difficultés nées de la guerre actuelle n'ont fait qu'aggraver les conditions, déjà mauvaises en temps normal, de notre approvisionnement en lait en nature. Le lait d'hier et d'aujourd'hui, de qualité insuffisante tant du point de vue hygiénique que du point, de vue énergétique, devait et doit ses graves défauts, d'une part aux inconvénients passagers dus à des eircoirstances éconorniques néfastes plus ou moins dürables, et, d'autre part, à une conception erronée, ou devenue fausse, des modalités mêmes de cet approvisionnement en lait.

\section{Le lait d'hier}

A. Le lait de consommation en nature se répartit en deux catégories : $1^{\circ}$ le lait cru produit et vendu sur place ; $2^{\circ}$ le lait industriel dont le transport à des distances variables nécessite un traitement comportant essentiellement le chauffage en vue d'une conservation de courte durée.

1. Le lait vendu cru est, en général, un lait très pollué et souvent pathogène. L'importance de la tuberculose bovine, l'abondance des mammites, la fréquence des affections intestinales - le tout sans aucune mesure restrictive en faveur de la production du lait - puis les méthodes défectueuses de traite et de manipulation expliquent aisément le lamentable état de choses que les hygiénistes ne cessent de déplorer en se heurtant à l'impassibilité des pouvoirs publics.

Une exception favorable concerne le lait de quelques étables urbaines ou suburbaines soumises à un contrôle sanitaire régulier, et tel est le cas, par exemple, des laitiers nourrisseurs du département de la Seine (2).

Evidemment, l'ébullition domestique évite bien des drames mais il en subsiste trop dont le lait est responsable ; l'ébullition, même correctement pratiquée, n'est qu'une sauvegarde, et le lait bouilli ne devient pas fatalement l'aliment parfait si nécessaire à l'enfant.

2. Le lait industriel est un lait de grand mélange rassemblé dans des centres de ramassage. Il est, dans ces centres, filtré, irrégulièrement chauffé et refroidi, puis transporté vers le centre de consommation. Il présente, au début, tous les défauts que nous

(1) Rapport présenté à la séance plénière du Comité |National de l'Enfance, Paris, 9 novembre 1944.

(2) G. Threutin. Le lait anonyme et le lait de qualité. Bull. Académie de Médecine, CXXVI, 1942, 208. 
venons de mentionner pour le lait cru et, à la fin, il aura subi de telles recontaminations que la pasteurisation effectuée, à supposer qu'elle ait été rationnelle, se révèlera, du point de vue de l'hygiène, absolument illusoire. Elle aura permis tout au plus une prolongation de la vie commerciale du lait traité.

Pour ajouter à cet état de fait, n'a-t-on pas vu des textes officiels recommander la javellisation des bidons à lait et oublier d'en prescrire le rinçage, se contentant, par ailleurs, de conseiller une réfrigération très relative "si possible au-dessous de $+12^{\circ}$. ».

Cette situation déplorable s'est encore aggravée avec les rigueurs de la guerre, particulièrement depuis 3 ans. Le ralentissement progressif des transports, le manque de combustible pour le chauffage du lait, les moindres possibilités de la réfrigération, l'addition de carbonate et de bi-carbonate de soude "facteur de putréfaction ", le non renouvellement et le mauvais entretien de la poterie, la raréfaction des produits de nettoyage, se sont ajoutés à la création du lait «standard» (1).

Ce lait standard est, comme chacun sait, un lait partiellement écrémé, résultant d'un mélange de lait entier et de lait écrémé : il est, du point de vue énergétique, très insuffisant; il est, du point de vue de l'hygiène, en proie à d'abondantes souillures liées à des manipulations supplémentaires; il constitue enfin, du point de vue économique et social, un nivellement par le bas, et c'est le moins qu'on en puisse dire.

Le lait industriel comprend encore le lait stérilisé, actuellement distribué en flacons de verre munis d'une fermeture canette. Ce lait, homogénéisé avant stérilisation, est souvent trop fortement chauffé et possède ainsi, par défaut technique, une saveur peu agréable ; mais, surtout, le mode d'obturation du récipient, particulièrement si ce dernier n'est pas d'étroite ouverture, permet des contaminations par rentrée d'air. L'expérience a confirmé que le lait stérilisé, ainsi conditionné, était de conservation précaire.

B. Il convient de faire une place à part aux laits concentrés et au lait sec. Malgré les techniques modernes de préparation, donnant toute satisfaction, ces laits conservés, modifiés, ne sont pas encore, en France, appréciés à leur juste valeur. Généralement, leur emploi s'effectue sur indication médicale, et les temps difficiles que nous continuons de vivre ont souligné l'intérêt exceptionnel de ces aliments. Malgré tout, jusqu'à présent dans l'esprit du public, le lait concentré et le lait sec ne sont encore que des laits de remplacement.

(1) Décret du 4 décembre 1941, fixant à 30 grammes par litre la teneur en matière grasse des laits pasteurisés. 
C. Réglementation. - Nous en tenant à deux textes essentiels qui, à eux seuls, pourraient modifier profondément la situation, nous citerons :

1. Le décret du 24 janvier 1934 (1), sur l'utilisation des laits provenant d'animaux atteints de tuberculose.

Ce décret prescrit la destruction de certains laits, trop fortement contaminés pour pouvoir être assainis, et la pasteurisation (réelle) des laits infectés à un faible degré ou suspects de l'être.

Ce décret est resté lettre morte.

2. Le décret du 26 avril 1939 (2), sur le contrôle des ateliers de pasteurisation.

Ce décret, non encore appliqué, fixe lai qualité légale du lait pasteurisé courant, vendu "ouvert», et celle du lait pasteurisé, " certifié » vendu en flacons cachetés.

L'écart entre la qualité légale, fixée par ce décret, et la qualité réelle, constatée par de très nombreux examens (3), est flagrant. Quant au lait pasteurisé "certifié », il fut l'objet, avant cette guerre, à Paris, d'une réalisation industrielle dont le succès eut été, pensonsnous, durable, s'il avait bénéficié d'un simple appui moral des autorités officielles.

\section{Le lait de demain}

La conclusion logique de la très fragmentaire étude critique que nous venons de présenter suffirait à dicter la politíque à suivre. Cependant, nous pensons que, dépassant les faits énoncés, il est nécessaire d'envisager, en matière d'approvisionnement en lait de l'agglomération parisienne, une conception plus générale, quelque peu différente de celle qui, pouvant sembler classique, fut jusqu'alors retenue.

Indépendamment de la nécessité, maintenant admise, d'une pasteurisation effective de la presque totalité du lait fourni en nature, deux considérations nous paraissent devoir être retenues :

1. Après sa pasteurisation, le lait est toujours, et abondamment, contaminé; et tout le bénéfice hygiénique de l'opération de chauffage est, de ce fait, perdu si le lait n'est pas protégé efficacement. Le seul moyen de le bien protéger est de le répartir en flacons stériles dès sa réfrigération consécutive à l'action thermique. Cette mise en flacons, effectuée à plusieurs dizaines de kilomèters du centre de consommation,

(1) Décret pris en application de la loi du 7 juillet 1933, sur la prophylaxie de la tuberculose bovine.

(2) Décret pris en application de la loi du 2 juillet 1935, sur l'assainissement du marché du lait.

(3) G. Threvins. Garantie hygiénique des laits pasteurisés. Bull. Académie de Médecine, CXXV, 1941, 400. 
serait un non-sens économique, étant donné l'important transport de poids mort qui en serait la conséquence, à l'aller et au retour. D'où l'intérêt de la pasteurisation effectuée au lieu même de consommation et, ce faisant, l'intervention précoce d'une réfrigération suffisante $\left(+2 \grave{a}+4^{\circ} C\right.$.) pour stabiliser le lait cru, nécessairement frais et propre, au cours de son transport avant chauffage.

2. La forte teneur du lait en eau (900 gr. par litre), la fragitité de ce lait et les risques de sa manutention font penser, en matière de transport rationnel, à l'avantage qu'il y aurait à fabriquer, aux lieux de production, des laits concentrés et des laits secs.

La concentration nécessite, du point de vue technique, un lait "non altéré", et cette sélection "obligée " est en faveur de l'hygiène. Le lait traité sur place ne subit ni brassage ni manipulations néfastes. Régionalement produit et "stabilisé», it peut permettre une qualité sensiblement constante quant au tuux de matière grasse, et l'importance diététique de la connaissance de sa valeur énergétique n'est pas à établir ici. On arriverait ainsi à une normalisation de la vente de ce produit (1), le prix pouvant être proportionnel à la valeur alimentaire. La facilité du stockage et du transport, la sûreté de l'emploi, sont d'autres arguments mieux connus.

Tout ceci étant posé, quelle politique suivre?

A. Amélioration générale de la production. - Cette amélioration générale n'a pas cessé de provoquer des développements pour le moins inutiles, mais elle peut être effective par l'application des deux textes fondamentaux dont nous avons précédemment parlé et par l'adoption de quelques mesures indiscutables :

1. Application de la loi du 7 juillet 1933 et du décret du 24 janvier 1934. Ces textes rendent possibles l'aménagement de l'habitat animal, l'élimination des animaux de l'espèce bovine atteints de formes de tuberculose particulièrement graves (contagieuse) et servent, simultanément, l'hygiène du lait et la valorisation du cheptel national.

2. Organisation de la lutte contre les mammites et, en particulier, contre les mammites streptococciques. L'expérience nous a montré que des résultats très intéressants pouvaient être obtenus (2) et. que leur généralisation n'est pas une utopie.

3. Encouragement des étables urbaines et suburbaines au moyen de primes de production contre de réelles exigences sanitaires ;

(1) La réglementation française actuelle concernant les laits concentrés et les laits secs assure déjà d'importantes garanties. Cette réglementation pourrait encore être améliorée. (A. TAPERNoux. Le Lait, XXIII, 1943, 1.)

(2) R. Gely et G. ThIeULin. L'Inspection sanitaire du lait à l'étable en vue de la prophylaxie et du traitement des mammites. Bull. de l'Académie Vétérinaire, XVI, 943, n $^{\circ} 11$. 
de semblables primes cessant d'être, comme précédemment, attribuées sans discrimination de qualité.

B. Diététique urbaine. - En France, le cas le plus difficile, par l'ampleur de la fourniture, est précisément celui de l'agglomération parisienne (1.200.000 litres de lait en nature, par jour, en 1939).

Pour donner au problème, depuis si longtemps posé, une solution valable, nous dirons, sans plus tergiverser, que, en dehors d'un lait cru de qualité, produit par les étables urbaines et suburbaines pour une petite minorité des besoins de la population, deux catégories de lait donneront, seules, la garantie indispensable, sous contrôle des services officiels compétents :

$1^{0}$ Le lait pasteurisé "certifié" (flacons cachetés);

$2^{\circ}$ Le lait concentré (non sucré, homogénéisé, stérilisé), et le lait sec.

L'actuel lait standard étant, sans délai, supprimé, il faut cesser de compter sur les laits pasteurisés ordinaires qui, quelquefois véritablement pasteurisés mais toujours recontaminés sont sans garantie et le resteront.

Ce lait pasteurisé courant ne peut pas être amélioré car les défauts qu'il présente sont inhẻrents aux conditions mêmes de son obtention ; améliorer ce lait, c'est tout simplement le transformer en lait pasteurisé "certifié ".

Le système de la eatégorisation des qualités (grades dans les pays anglo-saxons) n'est valable que si les producteurs et les consommateurs sont soucieux d'hygiène alimentaire et amateurs de la qualité correspondante.

A un autre point de vue il est désirable que le lait pasteurisé "certifié " porte 'l'indication, non seulement de son origine (centre de pasteurisation) et de la date de cette pasteurisation, mais encore de sa teneur en matière grasse. Il serait simple que, par exemple, une bande de papier vert ou un cachet sur les flacons indiquât une teneur supérieure à 35 grammes de matière grasse par litre. Ceci permettrait de reconnaître le lait possédant une valeur alimentaire supérieure et de le désigner pour certains usages ; son prix de vente étant plus élevé et toute fraude étant facilement décelable.

Les mêmes précisions sont exigibles du lait concentré : la boîte peut porter, non seulement la date de la fabrication et la quantité d'eau de dilution a ajouter pour reconstituer le lait originel, mais encore la teneur en matière grasse que possédera çe lait reconstitué.

Pour le lait sec, on admet actuellement deux catégories : 12 et $24 \%$ de matière grasse. 
Pour réaliser ee programme rationnel, il est indispensable :

10 D'avertir le Corps médical et d'éduquer les consommateurs ;

$2^{\circ} \mathrm{D}$ a favoriser, par une réclame officielle, les laits à recommander, et de condamner, dans une même propagande, les laits suspects c'est-à-dire dangereux ;

$3^{\circ}$ D'admettre un prix de vente plus élevé, pour les laits pasteurisés "certifiés", que pour les autres qui sont de mauvaise qualité ;

$4^{\circ}$ D'utiliser ces laits pasteurisés certifiẻs dans les centres hospitaliers et dans les œuvres d'assistance, conjointement avec le lait concentré non sucré et le lait sec.

\title{
Conclusion
}

Si l'on veut cesser d'exercer un contrôle hygiénique de pure forme, il faut admettre d'abord la sanction économique : prix de vente plus élevé pour les laits présentant toute garantie et doués d'une valeur énergétique supérieure.

Ces laits "meilleurs" doivent bénéficier d'une réclame officielle impartiale, les autres devant être publiquement condamnés - sous la responsabilité des Services officiels de contrôle.

Il est capital d'instruire tous les intéressés sur la valeur du lait pasteurisé "certifié » et sur celle du lait concentré non sucré et du lait sec.

D'une façon générale, l'amélioration de la qualité hygiénique des laits de consommation doit s'appuyer sur l'application des règlements actuels, et en particulier, sur celle du décret du 24 janvier 1934 et du décret du 26 avril 1939 - ce dernier nécessitant toutefois quelques modifications relatives à des confusions ou à son excessive souplesse aux dépens de l'hygiène publique.

L'approvisionnement en lait en nature, des Centres urbains, doit faire l'objet d'une politique rationnelle conditionnée par les raisons de l'hygiène et une Economie basée sur les techniques modernes.

\section{DOSAGES CO MPARATIFS DES DERIVÉS SULFAIMIDES DANS LE SANG ET LE LAIT DES NOURRICES ET DANS LES URINES DES NOURRISSONS (1)}

\author{
psr
}

BEDRINE, POITEAU et BISERTE

Le passage des sulfamides dans le lait des nourrices a été surtout étudié à l'Etranger. Les opinions sont divergentes et les résultats

(1) Travail de la Clinique Obstétricale de la Faculté de Médecine de Lille (Profes. suer H. PAUCOT); in Gynécologie et Obstétrique, XLIII, 1943, 154. 